\title{
Note
}

\section{Separation of the subunits of crotoxin by high-performance liquid chromatography}

\section{SHIH-HSIUNG WU}

Institute of Biological Chemistry, Academia Sinica, P.O. Box 23-106, Taipei (Taiwan)

FU-HSIUNG CHANG

Institute of Biochemical Sciences, National Taiwan University, Taipei (Taiwan)

and

MU-CHIN TZENG*

Institute of Biological Chemistry, Academia Sinica, P.O. Box 23-106, Taipei (Taiwan)

(Received November 24th, 1982)

Crotoxin is the major toxin in the venom of the Brazilian rattlesnake (Crotalus durissus terrificus) ${ }^{1}$. It is a complex of two subunits associated non-covalently: a basic protein (crotoxin PLA or crotoxin B) which carries the phospholipase (PLA) activity and an acidic protein (crotapotin or crotoxin $A$ ) which has no enzymic activity and is not toxic ${ }^{2,3}$. The lethal effect of crotoxin has been attributed to a pre-synaptic blockage of the neuromuscular transmission ${ }^{4}$, but it has been shown that the toxin acts also at the post-synaptic level ${ }^{5,6}$. Habermann and Breithaupt ${ }^{7}$ stated that the scparation of these two subunits was one of the three important goals in the study of crotoxin. In previous reports, these two subunits were separated after alkylation or acylation of the amino groups ${ }^{8}$, on a carboxymethylcellulose column at $\mathrm{pH} 4$ or on a DEAE-cellulose column in $6 M$ urea $^{2,3,9}$. More recently, two subunits of human pituitary thyrotropin and pike eel gonadotropin have been successfully separated by hydrophobic interaction chromatography ${ }^{10,11}$. Judging from the amino acid compositions ${ }^{12}$, the two subunits of crotoxin have a large difference in hydrophobicity and thus may be separated by reversed-phase liquid chromatography. In this work the separation of the two subunits of crotoxin by high-performance liquid chromatography (HPLC) was investigated, and an unexpected finding is reported.

\section{EXPERIMENTAL}

Crotoxin was isolated from the crude venom of Crotalus durissus terrificus (Sigma, U.S.A.) according to the method of Slotta and Fraenkel-Conrat ${ }^{1}$. After isoelectric precipitation, the precipitate was dissolved in acidified water $(\mathrm{pH} 3)$ containing $0.1 M$ sodium chloride and further purified by Sephadex G-50 (Pharmacia, Sweden) column chromatography. The purified crotoxin was homogeneous as tested by SDS-polyacrylamide gel electrophoresis. Crotapotin and crotoxin PLA were obtained by CM-Sephadex C-25 (Pharmacia) column chromatography of crotoxin with a linear gradient of 0.1-3.0 M ammonium formate (Ferak, G.F.R.) buffer (pH 3.5) ${ }^{2}$. 
For HPLC experiments, a $\mu$ Bondapak-CN column (Waters Assoc., U.S.A.) was used. The HPLC system consisted of two Waters Model 6000 pumps, a Waters UK-6 valve-loop injector, a Waters Model 450 variable-wavelength UV detector, a Waters Model 660 solvent programmer and a Houston Omniscribe two-channel chart recorder. Samples of about 15-30 $\mu \mathrm{g}$ were injected using a Microliter 802 syringe (Hamilton, Reno, NV, U.S.A.). Elution was carried out with a $0.05 \mathrm{M}$ solution of sodium phosphate (Merck, G.F.R.) and $0.05 \mathrm{M}$ sodium sulphate (Merck) buffer (pH 2.5 ), containing a linear gradient of acetonitrile (Alps, Taiwan) changing from $20 \%$ to $27.5 \%(\mathrm{v} / \mathrm{v})$ during the first $20 \mathrm{~min}$. The pressure was 1000 p.s.i. and the flow-rate was $1.5 \mathrm{ml} / \mathrm{min}$. The eluate was monitored at $230 \mathrm{~nm}$. Water for HPLC was prepared with a Milli-Q water purification system (Millipore, Bedford, MA, U.S.A.).

\section{RESULTS AND DISCUSSION}

The native crotoxin was separated into three peaks in the HPLC profile (Fig. 1). The first two peaks were crotapotin, as they had the same retention times as the purified compound (Fig. 2). This result is consistent with the results of Breithaupt et al. ${ }^{12}$, who reported that there were two variants of crotapotins. The third peak showed PLA activity and also could combine with the first two peaks to restore the toxicity to a level similar to that of native crotoxin. The purified crotoxin PLA, which has been demonstrated to be homogeneous by disc and SDS-polyacrylamide gel electrophoresis, surprisingly gave three peaks in HPLC (Fig. 3), with the smallest peak (the third in Fig. 3) having the same retention time as that of the third peak of native crotoxin in Fig. 1. When the crotoxin PLA purified from native crotoxin by HPLC was stored in the elution buffer overnight at room temperature, it still gave a single,
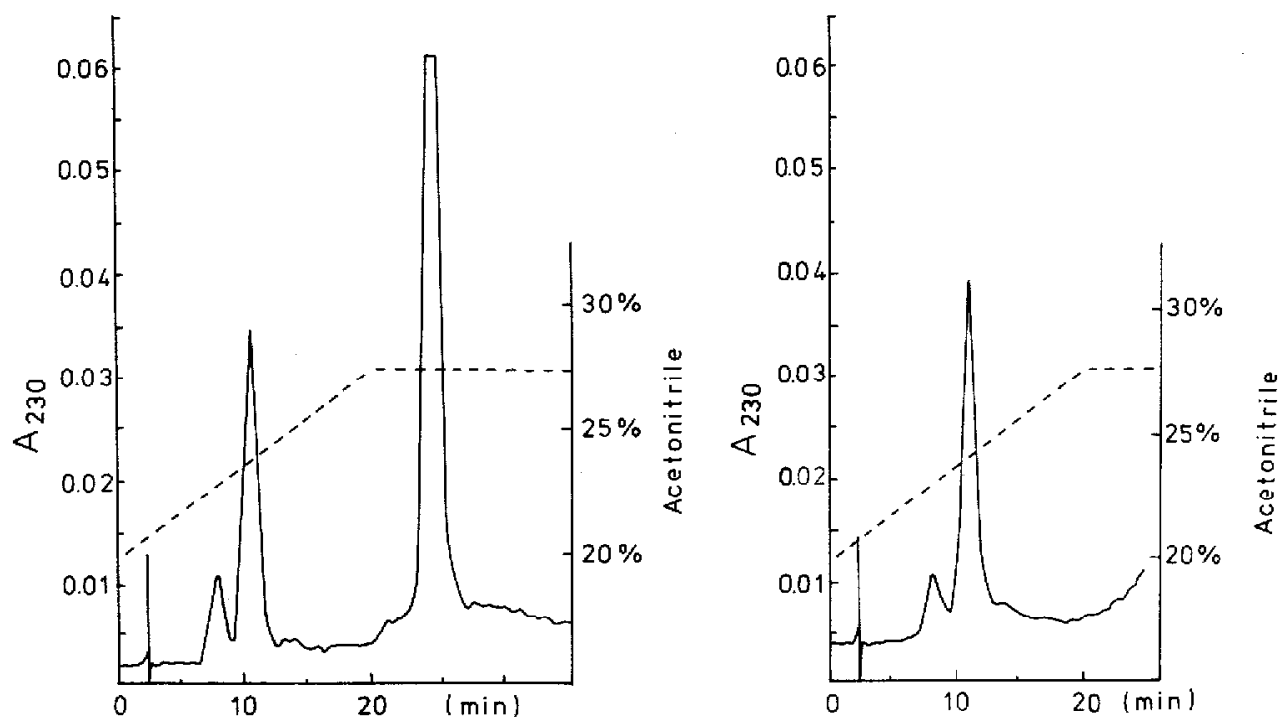

Fig. 1. HPLC of $30 \mu \mathrm{g}$ of native crotoxin.

Fig. 2. HPLC of $15 \mu \mathrm{g}$ of crotapotin. 

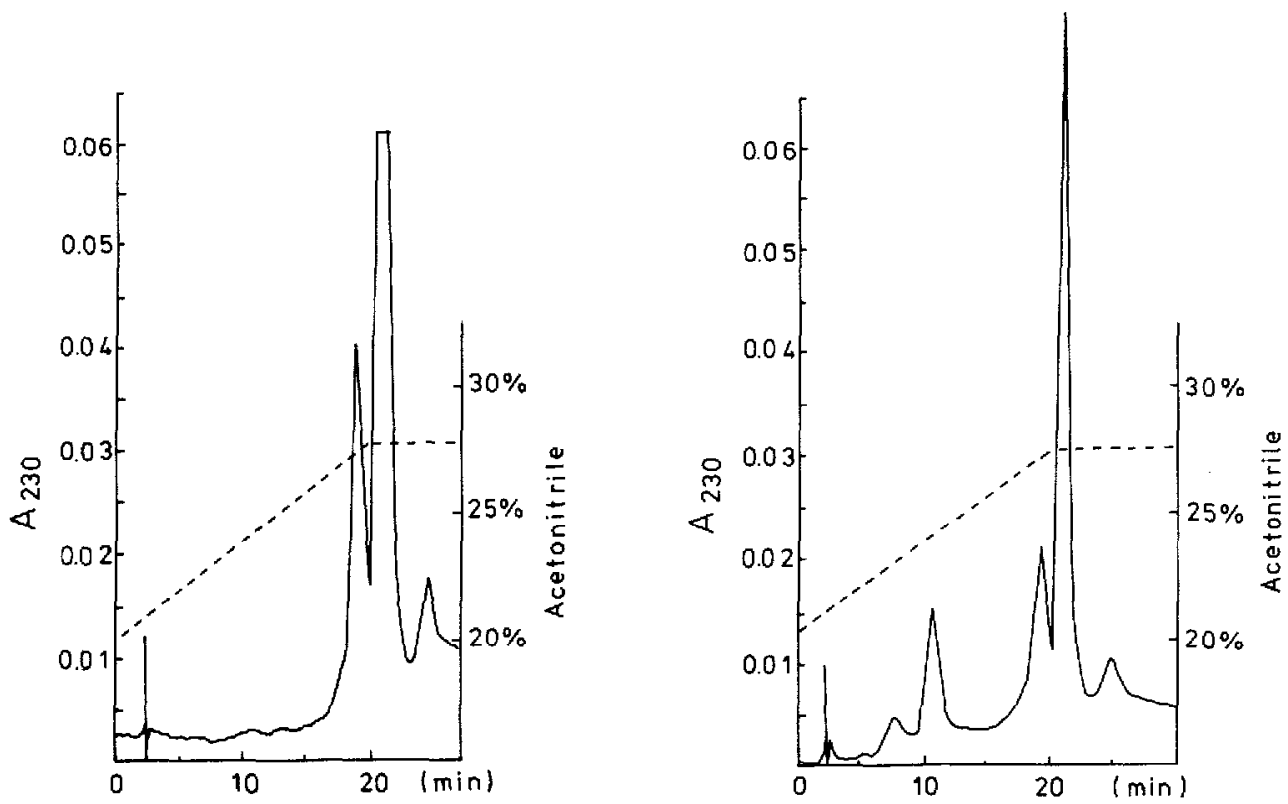

Fig. 3. HPLC of $30 \mu \mathrm{g}$ of crotoxin PLA.

Fig. 4. HPLC of $15 \mu \mathrm{g}$ of reconstituted crotoxin.

symmetrical peak in the HPLC profile with no change in the retention time; however when $3 M$ ammomium formate solution was added to this preparation for a few hours, the HPLC profile changed to one like that in Fig. 3. The crotoxin reconstituted from cortapotin and crotoxin PLA purified by CM-Sephadex chromatography did not give an HPLC profile (Fig. 4) similar to that of native crotoxin (Fig. 1), although the biological activity was restored.

The results showed that the dissociation of crotoxin by ion-exchange chromatography produced some irreversible changes in the crotoxin PLA subunit; nevertheless, this change did not influence the restoration of biological activity. The cause was traced to the ammonium formate buffer used, which somehow changed the hydrophobicity of crotoxin PLA. From these experiments, it appears that HPLC is a better method for separating crotoxin into its subunits.

\section{REFERENCES}

1 K. H. Slotta and H. Fraenkel-Conrat, Ber. Deut. Chem. Ges., 71 (1938) 1076.

2 K. Rübsamen, H. Breithaupt and E. Habermann, Naunyn Schmiedeberg's Arch. Exp. Pathol. Pharmakol., 270 (1971) 274.

3 R. A. Hendon and H. Fraenkel-Conrat, Proc. Nat. Acad. Sci. U.S., 68 (1971) 1560.

4 C. C. Chang and J. D. Lee, Naunyn-Schmiedeberg's Arch. Exp. Paihol. Pharmakol., 296 (1977) 159.

5 O. Vital-Brazil, Mem Inst. Butantan, Sao Paulo,33 (1966) 981.

6 C. Bon, J. P. Changeux, T. W. Jeng and H. Fraenkel-Conrat, Eur. J. Biochem., 99 (1979) 471.

7 E. Habermann and H.Breithaupt, Toxicon, 16 (1978) 19.

8 H. Fraenkel-Conrat and -B. Singer, Arch. Biochem. Biophys., 60 (1956) 64.

9 R. Hendon, D. Roy and H. Fraenkel-Conrat, Toxicon, 8 (1970) 135.

10 G. Jacobson, P. Roos and L. Wide, Biochim. Biophys. Acta, 536 (1978) 363.

11 T. B. Lo, F. L. Huang and G. D. Chang, J. Chromatogr., 215 (1981) 229.

12 H. Breithaupt, K. Rübsamen and E. Habermann, Eur. J. Biochem., 49 (1974) 333. 